\title{
Atitudes em relação à informação de preços de alimentos
}

\author{
Etiénne Groot ${ }^{1}$ \\ ${ }^{1}$ Universidade Estadual Paulista "Júlio de Mesquita Filho" - UNESP, Campus de Dracena, Dracena, São Paulo, Brasil. \\ E-mail: etigroot@dracena.unesp.br
}

Recebido: 28/04/2017; Aceito: 19/07/2017

\section{RESUMO}

O Brasil passa por um período com tendência inflacionária e, desta forma, as alterações de preços repercutem no comportamento dos consumidores. Objetivou-se com o presente estudo a avaliação da percepção dos consumidores em relação aos preços dos alimentos, a sua atitude em buscar os menores preços de mercado e a sua propensão a utilizar informações de mídia eletrônica. Para isso, foram consideradas 54 entrevistas pessoais realizadas com consumidores de Dracena/SP, com auxílio de questionário estruturado. Os resultados mostram que, mesmo em ambiente inflacionário, a percepção dos consumidores em relação ao conhecimento dos preços dos produtos ainda é alta, principalmente entre as pessoas com mais de 45 anos de idade. Muitos consumidores pesquisam os preços dos produtos para planejarem as suas compras. Os consumidores com mais de 45 anos de idade são os menos propensos em utilizar informação dos meios eletrônicos.

Palavras-chave: Dracena, consumidor, pesquisa de preços

\section{Attitudes toward food prices}

\begin{abstract}
Brazil is going through a period with inflationary trend and then prices changes affect consumer behavior. It was aimed with the present study to evaluate the consumers' perceptions regarding food prices, their attitude in seeking the best market prices and their propensity to make use of electronic media information. Thus, it was considered 54 personal interviews carried out with consumers from Dracena, State of Sao Paulo, with the aid of a structured questionnaire. The results show that, even in an inflation environment, consumers' perception about product prices is still high, especially among people over 45 years old. Many consumers search product prices to plan their purchases. Consumers over 45 years old are less likely to use electronic media information.
\end{abstract}

Key words: Dracena, consumer, price survey. 
O momento econômico brasileiro é de incertezas. Segundo o Banco Central do Brasil (BACEN, 2016), a meta estipulada para a inflação pelo Conselho Monetário Nacional era de 4,5\% no ano 2016. Entretanto, a variação acumulada dos últimos 12 meses do IPCA em julho de 2016, publicado pelo Instituto Brasileiro de Geografia e Estatística (IBGE, 2016), foi de 9,56\%. O maior valor desde dezembro de 2003. Segundo a Pesquisa de Orçamento Familiar (POF) de 2008-2009 (IBGE, 2010), os gastos com alimentação correspondem a $16,1 \%$ da despesa familiar total e o setor de alimentação tem contribuído significativamente para o aumento da inflação.

Ainda que não seja possível eliminar a alta da inflação, é possível realizar pesquisas de menor preço no intuito de reduzir custos, podendo evitar gastos desnecessários por parte do consumidor. Em 2016, de acordo com $12^{\circ}$ levantamento anual de preços dos supermercados brasileiros, realizado pela Associação Brasileira de Defesa do Consumidor (PROTESTE, 2016), a economia anual com a compra da Cesta Básica 2 - voltada para quem prioriza apenas os preços baixos, em estabelecimentos com preços mais competitivos foi estimada em R\$ 2.271,02, para a cidade de São Paulo.

Embora os benefícios da pesquisa de preços sejam evidentes, expressiva parcela da população não a realiza. Uma das razões é a falta de disponibilidade de tempo para esta atividade (HONG; SHUM, 2006) e outra é a capacidade de pesquisar preços (GAURI et al., 2008). Além destes custos pela busca da informação, é possível ainda que outros fatores influenciem na disposição pela busca de informação de preços. Segundo Urbany et al. (1996), além de considerarem os custos e os benefícios (economia) da busca de informações, os consumidores ainda avaliam o seu conhecimento dos preços, hábitos e retorno social. Uma vez adquirido a percepção do padrão de preços dos estabelecimentos comerciais, os consumidores mantêm o status quo de suas compras até perceberem mudanças na relação beneficio/custo da busca da informação. Esta ruptura pode ocorrer quando a população recebe noticias de variações significativas dos preços praticados em diferentes estabelecimentos comerciais, promoções, abertura de novos estabelecimentos ou ainda a percepção de inflação.

Visando eliminar ou reduzir a limitação imposta pelos custos de busca de informação de preços, hoje existem no Brasil diversas instituições, de fins não comerciais, que realizam pesquisas de preços de produtos alimentícios em diferentes estabelecimentos comerciais e disponibilizam os resultados de forma acessível e barata aos consumidores. Por exemplo, a Faculdade de Ciências Agrárias e Tecnológicas (FCAT/Unesp) desenvolve projetos de extensão universitária que pesquisam e divulgam preços de produtos alimentícios aos consumidores de Dracena (UNESP, 2016).

Diante deste contexto, objetivou-se avaliar as atitudes dos consumidores de Dracena, município do oeste paulista, em relação aos preços dos alimentos.

As percepções e atitudes dos consumidores foram avaliadas através de entrevistas pessoais. Para auxílio às entrevistas desenvolveu-se um questionário estruturado, contendo questões que permitem ao consumidor expor sua percepção em relação ao conhecimento dos preços dos alimentos, a atitude de pesquisar preços de produtos antes de realizar as compras e propensão ao uso de informação de preços no planejamento das compras. As percepções foram mensuradas por meio de escala de Likert, com escala de 4 níveis, variando de "concordo totalmente" a "discordo totalmente". O perfil sociodemográfico do entrevistado também foi obtido nas entrevistas.

As entrevistas ocorreram durante o mês de junho de 2016. Os entrevistadores, alunos da graduação da FCAT/Unesp realizaram as entrevistas com a orientação e supervisão de um docente da faculdade.

As entrevistas ocorreram na entrada de um supermercado e nas próprias casas dos consumidores, no bairro Palmeiras, em Dracena/SP. No total, entrevistou-se 59 consumidores, o que resultou em 54 questionários válidos. Para o cálculo do erro amostral considerou-se as orientações de Luchesa e Chaves Neto (2011). Assim, com um grau de confiança de $95 \%$ e expectativa da verdadeira proporção de um dos níveis da variável escolhida de $50 \%$, o erro amostral foi calculado em 13,6\%. Tratando-se de pesquisa exploratória, este nível de erro pode ser admitido, porém os resultados não podem ser generalizados a toda população. Segundo Chernyak e Nebukin (sd), a pesquisa exploratória é realizada no estágio inicial do projeto e ajuda na obtenção de uma visão mais ampla da essência do problema, o que contribui na formulação de ideias e na proposição de hipóteses em futuros trabalhos.

As características sócio-demográficas dos consumidores que participaram da pesquisa podem ser vistas na Tabela 1. A faixa etária mais presente entre os consumidores entrevistados é de 30 e 65 anos de idade. Os consumidores com menos de 45 anos representam $51,9 \%$ de todos os consumidores. As mulheres participaram mais no presente estudo que os homens. Este fato é desejável uma vez que são elas as grandes responsáveis pelas decisões de compras da família. Nos dias atuais, além de contar com a própria renda, as mulheres são responsáveis pelo controle do orçamento familiar. Além de maiores consumidoras, as mulheres exercem expressivas influencias sobre o comportamento de compra dos homens. Estima-se que as mulheres sejam as responsáveis por $80 \%$ das decisões de compra 
da família, especialmente no que se refere a alimentos (FIORI, 2011).

Em referência ao nível de estudos dos consumidores entrevistados, $29,6 \%$ disseram ter frequentado a universidade, ou seja, possuem o estudo superior, completo ou incompleto. Por outro lado, 22,2\% possuem ensino fundamental, completo ou incompleto. De acordo com o Censo Demográfico de 2010 (IBGE, 2011), 36,5\% da população da cidade possuíam estudos superiores, completo ou incompleto. Contrastando estas informações observa-se que este tipo de consumidor está sub-representado na presente pesquisa.

O tamanho da família, muitas vezes avaliado pela densidade familiar, influencia em sua própria renda per capita e esta acaba determinando, em grande medida, o comportamento de consumo das pessoas que realizam as compras. $\mathrm{Na}$ amostragem, 40,7\% dos entrevistados vivem em residências com mais de 3 pessoas enquanto que 7,4\% moram em residências com apenas 1 morador. Esses números mostram que a densidade domiciliar dos entrevistados é superior à da população. Em Dracena, $38,1 \%$ dos lares reside apenas 1 pessoa e em $1,5 \%$ dos lares residem mais de 3 pessoas (IBGE, 2011).

Os resultados gerais da autoavaliação dos consumidores sobre os seus conhecimentos de preços de alimentos (variável “conhecimento"), ao hábito de realizar pesquisa em diferentes estabelecimentos antes das compras (variável "pesquisa") e a sua propensão em consultar fontes de informação de preços de produtos em páginas de internet (variável "informação”), estão representados na Figura 1.

Praticamente, 1/3 dos consumidores entrevistados se mostraram totalmente de acordo que possuem ótimo conhecimento dos preços dos produtos. Pouco mais de
$40 \%$ dos consumidores afirmaram estar de acordo que possuem ótimo conhecimento dos preços dos produtos e pouco mais de $1 / 4$ dos entrevistados discordaram em ter ótimo conhecimento dos preços dos produtos. Comparando estes resultados com os obtidos por Silva e Urdan (2008), que observaram que $1 / 3$ dos consumidores cometem desvios de até $25 \%$ entre o preço real e o preço percebido, é possível que os consumidores de Dracena estejam superestimando os seus conhecimentos em relação aos preços dos alimentos.

A pesquisa de preço é a forma mais comum de busca de informação para tomar as decisões de compras. Indagados sobre o assunto, 38,9\% dos entrevistados se disseram totalmente de acordo com a afirmação que sempre fazem pesquisa de preço em diferentes estabelecimentos comerciais antes das compras e $22,2 \%$ se disseram de acordo, ou seja, em maior ou menos medida, $61,1 \%$ dos consumidores realizam pesquisas dos preços em Dracena antes de suas compras.

Embora seja fundamental, o que limita a pesquisa de preço pelos consumidores é o custo de oportunidade do tempo. Para que realizem a pesquisa de preço, uma das condições é que o custo de oportunidade do tempo seja menor que a percepção de ganho com a compra dos produtos a um preço menor. Os meios eletrônicos representam uma opção de pesquisa de preços.

Nas entrevistas, $77,8 \%$ dos consumidores concordaram totalmente e outros $5,5 \%$ concordaram que, caso existisse informações dos preços dos produtos de Dracena num site, sempre fariam consultas para planejar suas compras. Estes resultados mostram que as pessoas preferem estar bem informadas para tomar as suas decisões. Qualquer iniciativa neste sentido estará melhorando o bem-estar das pessoas.

Tabela 1. Características sócio-demográficas dos consumidores entrevistados

\begin{tabular}{lcc}
\hline Idade & $\mathrm{N}^{\circ}$ pessoas & Porcentagem \\
\hline Menos de 30 anos & 7 & 13,0 \\
Mais de 9 e menos de 45 anos & 21 & 38,9 \\
Mais de 44 anos e menos de 65 anos & 17 & 31,5 \\
Mais de 64 anos & 9 & 16,7 \\
\hline Gênero & $\mathrm{N}^{\circ}$ pessoas & Porcentagem \\
\hline Masculino & 17 & 31,5 \\
Feminino & 37 & 68,5 \\
\hline Nível de estudo & $\mathrm{N}^{\circ}$ pessoas & Porcentagem \\
\hline Ensino fundamental, completo ou incompleto & 12 & 22,2 \\
Ensino médio, completo ou incompleto & 22 & 40,7 \\
Ensino superior, completo ou incompleto & 16 & 29,6 \\
Outros estudos & 4 & 7,4 \\
\hline Número de pessoas na casa & $\mathrm{N}^{\circ}$ pessoas & Porcentagem \\
\hline 1 Pessoa & 4 & 7,4 \\
2 Pessoa & 18 & 33,3 \\
3 Pessoa & 10 & 18,5 \\
Mais de 3 pessoas & 22 & 40,7 \\
\hline Total & 54 & 100,0 \\
\hline
\end{tabular}




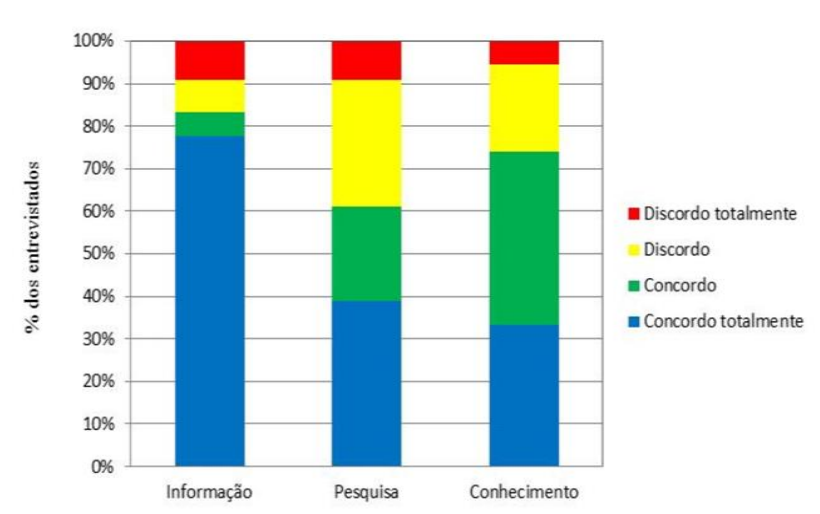

Figura 1. Grau de concordância em relação ao conhecimento de preços de produtos, pesquisa de mercado, substituições de produtos e uso de informação nas compras pelos consumidores de Dracena-SP

Além da divulgação tradicional dos preços dos produtos em sites, existem hoje aplicativos de celulares que comparam os preços de diferentes supermercados e planeja automaticamente a compra no sentido de minimizar os gastos e otimizar o tempo dos consumidores.

De acordo com o teste U de Mann Whitney, não existem diferenças significativas nas opiniões entre entrevistados de diferentes gêneros (masculino $\mathrm{x}$ feminino), nível de estudos (universitário $\mathrm{x}$ outros estudos), número de pessoas que vivem na casa (até 3 pessoas $\mathrm{x}$ mais de 3 pessoas). As únicas diferenças significativas, ao nível de significância de $10 \%$, foram observadas entre pessoas de diferentes idades (menos de 45 anos x mais de 44 anos). As pessoas das diferentes faixas etárias tiveram diferentes percepções sobre os seus conhecimentos de preços de alimentos e a respeito da disposição ao uso de informação.

As pessoas com mais de 44 anos sentem que estão mais informadas que as pessoas mais jovens e, caso houvesse a disponibilidade informações dos preços dos alimentos comercializados em Dracena num site, elas estariam menos dispostas a usar tais informações para planejamento as suas compras. Ou seja, as pessoas que se sentem mais informadas acabam buscando menos informação.

Há outra possível explicação para o menor interesse no uso de informações dos meios eletrônicos pelas pessoas com mais de 44 anos. Embora as pessoas mais idosas possuam maior dificuldade em seguir as constantes inovações tecnológicas, existem cada vez mais pessoas idosas interessadas em empregar a informática para aumentar o seu grau de socialização e usufruir dos benefícios propiciados por esta tecnologia (CARDOSO et al., 2014). Embora presente, esta limitação pode ser cada vez menor, principalmente com a existência de programas de inclusão digital de pessoas mais idosas.

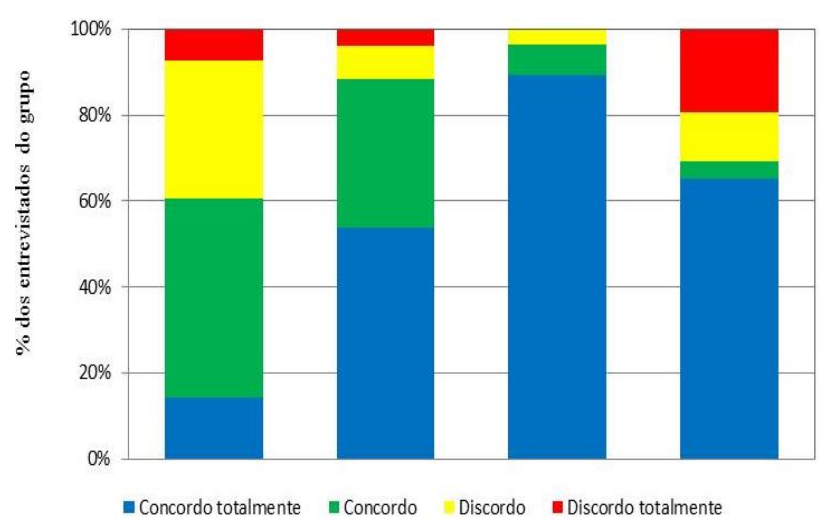

Figura 2. Grau de concordância em relação ao conhecimento de preços de produtos e uso de informação nas compras pelos consumidores de Dracena-SP, segundo a sua faixa etária

Conhecer os preços dos produtos é uma condição necessária para os consumidores racionalizar os seus gastos e, assim, aumentar o poder aquisitivo. $\mathrm{O}$ conhecimento dos produtos é adquirido com a experiência de compra dos consumidores, bem como pela pesquisa de preços. A pesquisa de preços pode ser feita pelo próprio consumidor e/ou por meio de uma organização, que logo divulga as informações à sociedade.

Na região estudada há uma parcela significativa de consumidores que consideram conhecer bem os preços dos produtos, caso esta percepção esteja equivocada, pode ter graves implicações nos gastos familiares.

$O$ grupo de pessoas que consideram ter conhecimento de preços pode não estar motivada em buscar novas informações, e por isso, qualquer programa que vise pesquisar e divulgar preços deve conscientizar o seu público alvo sobre o seu desconhecimento e, logo, a respeito das consequências da assimetria de informação.

O tempo tem valor e, por isso, o custo de oportunidade do tempo acaba limitando a pesquisa de mercado por parte de quase $40 \%$ da população da região ao realizar as compras de alimentos.

\section{Referências Bibliográficas}

BACEN. BANCO CENTRAL DO BRASIL. Histórico de metas para a inflação no Brasil. Brasília-DF: BACEN, 2016. Disponível em: http://www.bcb.gov.br/Pec/metas/TabelaMetaseResultados.pd f. Acesso em 07 de set. 2016.

CARDOSO, D.R.; STEFANELLO, K.V.B.; SOARES, K.V.B.C.; ALMEIDA, W.M. Os benefícios da informática na vida do idoso. Computer on the beach, Florianópolis-SC, v. 5, n. 1, p. 340-349, 2014. Disponível em: <http://siaiap32.univali.br/seer/index.php/acotb/article/view/5 338/2795>. Acesso 01 jun. 2017.

CHERNYAK, O.; NEBUKIN, V. Application of survey sampling methods to Market research. Shevchenko 
University, Ukraine. Disponível em: probability.univ.kiev.ua/school09/papers/Article_ENG_7_Che rnyak_Nebukin.doc. Acesso em: 03 de set. 2016.

DICKSON, P. R.; SAWYER, A. G. The price knowledge and search of supermarkets shoppers. Journal of Marketing, Birmingham-CA, v. 54, n. 3, p. 42- 53, 1990.

FIORI, D. Mulheres: um diagnóstico da participação feminina na economia Brasileira. Departamento de Sustentabilidade do Walmart Brasil. 2011. Disponível em: < http://www.ruscheleassociados.com.br/pdf/walmulheres.pdf >. Acesso em: 17 jul. 2017.

GAURI, D. K.; SUDHIR, K.; TALUKDAR, D. The temporal and spatial dimensions of price search: Insights from matching household survey and purchase data. Journal of Marketing Research, v. 45, n. 2, p. 226 - 240, 2008.

HONG, H.; SHUM, M. Using price distribution to estimate search costs. RAND Journal of Economics, v. 37, n. 2, p. $257-275,2006$.

IBGE. INSTITUTO BRASILEIRO DE GEOGRAFIA E ESTATÍSTICA. Sistema Nacional de Índices de Preços ao Consumidor: séries históricas, IPCA. Brasília-DF: IBGE, $2016 . \quad$ Disponível em: http://www.ibge.gov.br/home/estatistica/indicadores/precos/in pc_ipca/defaultseriesHist.shtm. Acesso em: 06 set. 2016.

IBGE. INSTITUTO BRASILEIRO DE GEOGRAFIA E ESTATÍSTICA Censo 2010. Brasília- DF: IBGE, 2011. Disponível em: http://www.censo2010.ibge.gov.br/. Acesso em: 06 set. 2016.
IBGE. INSTITUTO BRASILEIRO DE GEOGRAFIA E ESTATÍSTICA. Pesquisa de orçamentos familiares 20082009. Aquisição alimentar domiciliar per capita: Brasil e grandes regiões. Brasília-DF: IBGE, 2010. Disponível em: http://biblioteca.ibge.gov.br/visualizacao/livros/liv47307.pdf. Acesso em: 06 de set. 2016.

LUCHESA, C. J.; CHAVES NETO, A. Cálculo do tamanho da amostra nas pesquisas em Administração. Curitiba-PR: Unicuritiba, 2011.27 p.

PROTESTE. ASSOCIAÇÃO BRASILEIRA DE DEFESA DO CONSUMIDOR. Economia em supermercado pode chegar a até $\mathbf{R} \$ \mathbf{2 . 0 3 2 , 4 5}$ por ano em SP aponta pesquisa da PROTESTE. Rio de Janeiro-RJ: PROTESTE, 2016. Disponível em: https://www.proteste.org.br/institucional/imprensa/pressrelease/2016/economia-em-supermercado-pode-chegar-a-ater-203245-por-ano-em-sp-aponta-proteste. Acesso em: 26 maio 2017.

SILVA, M. A.; URDAN, A. T. O conhecimento do consumidor sobre preços: um exame de produtos de oito categorias de bens duráveis. Revista de Administração da Mackenzie, São Paulo-SP, v. 9, n. 2, p. 82-103, 2008.

UNESP. UNIVERSIDADE ESTADUAL PAULISTA. Projetos de extensão. 2016. Disponível em: http://www.dracena.unesp.br/\#!/extensao/projetos-deextensao1303/. Acesso em: 07 set. 2016.

URBANY, J. E.; DICKSON, P. R.; KALAPURAKAL, R. Price search in the retail grocery market. Journal of Marketing, Birmingham-CA, v. 60, n. 2, p. 91 - 104, 1996. 